\title{
Acupuntura como método alternativo de analgesia durante parto: uma revisão integrativa
}

\author{
Acupuncture as an alternative method of analgesia during delivery: an integrative review \\ La acupuntura como método alternativo de analgesia durante la entrega: una revisión integrativa
}

\begin{abstract}
RESUMO
Objetivo: Identificar se a acupuntura produz de fato alívio das dores do parto em nulíparas e multíparas. Método: Trata-se de um estudo de revisão integrativa em que foram utilizadas as bases de dados eletrônicas Pubmed e Medline, as quais foram acessadas por meio da Biblioteca Virtual em Saude. Além dessas, também foi utilizada a SciELO para a busca de artigos. Os Descritores em Ciência da Saúde (DeCS) foram "Analgesia por Acupuntura", "Trabalho de Parto" e "Paridade", bem como, com suas respectivas traduções para o inglês, publicados no período de 2003 a 2014 . A coleta de dados foi realizada em outubro de 2019. Resultados: Dos estudos examinados, somente um concluiu que não houve efeito analgésico sobre as dores do parto com o uso da acupuntura. Conclusão: A acupuntura demonstra potencial para contribuir no alívio das dores do trabalho de parto e presta um olhar holístico das parturientes.
\end{abstract}

DESCRITORES: Analgesia por acupuntura; Trabalho de Parto; Dor do Parto.

\section{ABSTRACT}

Objective: To identify whether acupuncture actually provides relief from labor pain in nulliparous and multiparous women. Method: This is an integrative review study in which the electronic databases Pubmed and Medline were used, which were accessed through the Virtual Health Library. In addition to these, SciELO was also used to search for articles. The Health Science Descriptors (DeCS) were "Analgesia for Acupuncture", "Labor and Delivery" and "Parity", as well as, with their respective translations into English, published in the period from 2003 to 2014. Data collection was conducted in October 2019. Results: Of the studies examined, only one concluded that there was no analgesic effect on labor pains with the use of acupuncture. Conclusion: Acupuncture shows the potential to contribute to the relief of labor pains and provides a holistic view of parturients.

DESCRIPTORS: Acupuncture analgesia; Childbirth Work; Labor Pain.

\section{RESUMEN}

Objetivo: identificar si la acupuntura realmente proporciona alivio del dolor de parto en mujeres nulíparas y multíparas. Método: Este es un estudio de revisión integrador en el que se utilizaron las bases de datos electrónicas Pubmed y Medline, a las que se accedió a través de la Biblioteca Virtual en Salud. Además de estos, SciELO también se utilizó para buscar artículos. Los Descriptores de Ciencias de la Salud (DeCS) fueron "Analgesia para la acupuntura", "Trabajo y parto" y "Paridad", así como, con sus respectivas traducciones al inglés, publicados en el período de 2003 a 2014. La recopilación de datos fue realizado en octubre de 2019. Resultados: de los estudios examinados, solo uno concluyó que no había efecto analgésico sobre los dolores de parto con el uso de la acupuntura. Conclusión: la acupuntura muestra el potencial de contribuir al alivio de los dolores de parto y proporciona una visión holística de las parturientas.

DESCRIPTORES: Analgesia de acupuntura; Trabajo de parto; Dolor de Parto.

RECEBIDO EM: 29/06/2020 APROVADO EM: 04/08/2020

\section{Tatiane Pombo}

Graduanda em Medicina, Centro Universitário de Maringá (UNICESUMAR).

ORCID: 0000-0002-7060-5019

\section{Ana Carla Cardoso Costa}

Graduanda em Medicina, Centro Universitário de Maringá (UNICESUMAR).

ORCID: 0000-0001-6567-0732 


\section{artigo}

Pombo, T.; Costa, A.C.C.; Ferreira, F.S.; Cardoso, S.M.; Ribeiro, R.; Mendes, T.P.L.C.; Antunes, M.B.;

Acupuntura como método alternativo de analgesia durante parto: uma revisão integrativa

\section{Felipe Sá Ferreira}

Médico. Mestrando em Saúde Pública pela Faculdade de Saúde Pública da Universidade de São Paulo (USP).

ORCID: 0000-0002-8987-5289

\section{Sarah Marques Cardoso}

Graduanda em Medicina, Centro Universitário de Maringá (UNICESUMAR).

ORCID: 0000-0003-1254-1464

\section{Rafaela Ribeiro}

Graduanda em Medicina, Centro Universitário de Maringá (UNICESUMAR).

ORCID: 0000-0002-3482-0758

\section{Thaís Pordeus Leite Costa Mendes}

Médica. Especialista em Urgência e Emergência pela Faculdade Metropolitana do Vale do Aço (FAMEV).

ORCID: 0000-0003-3969-5877

\section{Marcos Benatti Antunes}

Enfermeiro. Doutor em Enfermagem pela Universidade Estadual de Maringá (UEM). Docente do Curso de Medicina do Centro Universitário de Maringá (UNICESUMAR).

ORCID: 0000-0002-5139-8827

\section{INTRODUÇÃO}

A acupuntura, um dos métodos de tratamento da medicina tradicional chinesa, é amplamente aplicada para o alívio da dor ${ }^{(1)}$. Existem pontos no corpo que quando estimulados ativam os chamados "meridianos", canais por onde passa a força vital ou QI. A acupuntura baseia-se na estimulação desses meridianos, por meio de agulhas, esferas, eletricidade ou, até mesmo, sementes. Cada meridiano está associado diretamente com um sistema fisiológico e/ou mental do indivíduo. Dessa forma, a estimulação de tais pontos modifica a circulação da energia vital (QI) e, também, do fluxo sanguíneo (XUÈ) ${ }^{(2,3)}$.

Os mecanismos envolvidos na analgesia da dor pela acupuntura ainda não estão totalmente elucidados, porém muitas pesquisas mostraram que há liberação de peptídeos e opióides tanto no sistema nervoso central quanto no periférico. Além disso, resultados também revelaram que há alteração na função neuro-hormonal ${ }^{(4,5)}$.

O controle da dor do trabalho de parto (TP) tem sido um assunto polêmico entre os obstetras $^{(6,7)}$, mas há um interesse no desenvolvimento da acupuntura como opção aos analgésicos para tratar essa dor ${ }^{(1)}$. Segundo a Organização Mundial da Saúde (OMS), a taxa ideal de cesáreas deve estar entre 10\% e $15 \%$ e está associada a um decréscimo na mortalidade materna e neonata ${ }^{(8)}$. Porém, no Brasil mais da metade dos partos $(55,5 \%)$ realizados na rede pública são pela via cesária $^{(9)}$, e um dos principais motivos que levam a isso é o medo da dor do parto ${ }^{(6,7,9)}$.

A dor durante o TP que é produzida através de contrações uterinas, dilatação cervical e aumento do assoalho pélvico e vaginal é considerada um dos tipos de dor mais intensa ${ }^{(7)}$. Entretanto, a dor do trabalho de parto não depende somente do mecanismo fisiológico inato de cada mulher. Ela é também ligada às características individuais de cada paciente, como fatores socioculturais, socioeconômicos e emocionais. Para muitas, a dor é considerada muito sofrida e, até mesmo, aterrorizante, o que as fazem optar por métodos que aliviem esse sofrimento, através de analgésicos ou até mesmo submetendo-se a cesariana. Atentando-se para esses fatos, é de suma importância conhecer as experiências e expectativas de cada mulher em relação ao momento do parto ${ }^{(10,11)}$.

Um estudo que teve como objetivo identificar a percepção de parturientes sobre o uso da acupuntura na analgesia das dores do parto, concluiu que muitas mulheres têm interesse em experimentar esse método de controle da dor e assim conhecer outras formas de lidar com esse momento tão único na vida de cada mulher, o parto ${ }^{(12)}$.
Portanto, objetivou-se com o presente estudo identificar, por meio de uma revisão integrativa, se a acupuntura produz de fato alívio das dores do parto em nulíparas e multíparas. A partir disso, tornar esse conhecimento público de modo a transformar o processo do parto menos exaustivo para as gestantes e para os profissionais, encorajando as mulheres a optarem pelo parto natural.

\section{METODOLOGIA}

Trata-se de um estudo de revisão integrativa que possibilita a sistematização de resultados a partir de uma questão norteadora, permitindo a maximizar os conhecimentos de um objeto de estudo.

Foram utilizadas as bases de dados eletrônicas National Library of Medicine (Pubmed) e Medical Literatura Analysis and Retrieval System Online (Medline), as quais foram acessadas por meio da Biblioteca Virtual em Saude (BVS). Além dessas, também foi utilizada a Scientific Eletronic Library Online (SciELO) para a busca de artigos. A busca foi norteada pela questão: A acupuntura possui efeito analgésico sobre as dores do trabalho de parto?. A coleta de dados para responder esse questionamento foi realizada em outubro de 2019. Buscou-se artigos relacionados à analgesia e o alívio das dores do trabalho 
de parto independente da paridade das mulheres neles contemplados, nos idiomas inglês e português. As pesquisas foram realizadas com os Descritores em Ciência da Saúde (DeCS) "Analgesia por Acupuntura", "Trabalho de Parto" e "Paridade", bem como, com suas respectivas traduções para o inglês, "Acupuncture Analgesia", "Labor, Obstetric" e "Parity", correlacionando-as por meio dos operadores booleanos "e" ou "and" para formar as combinações.

Foram incluídos artigos que contemplassem os seguintes critérios: estudos randomizados, observacionais ou ensaios clínicos que contemplassem a prática $\mathrm{da}$ acupuntura, inclusive as suas principais variações, a acupressão, a eletroacupuntura e a moxabustão - acupuntura térmica, como método de alívio das dores sofridas por gestantes durante o trabalho de parto. A amostra foi composta por somente por artigos publicados no período de 2003 a 2014.

Foram excluídos artigos que não se tratavam de estudos randomizados, observacionais e ensaios clínicos. Além disso, foram descartados artigos que contemplavam outras terapias não farmacológicas para o alívio da dor do parto, que não fosse a acupuntura e suas principais variações (acupressão, eletroacupuntura e moxabustão - acupuntura térmica).

A análise dos artigos selecionados foi realizada por dois avaliadores, TAP e ACC, sendo essa feita de forma individual. Nos casos em que não houve consenso sobre a inclusão, um terceiro autor, SMC, foi responsável pela avaliação de tal artigo.

Para selecionar os artigos, primeiramente eles foram identificados e em seguida foram aplicados os critérios de inclusão e exclusão para saber a elegibilidade dos artigos. Por fim, foi concluída a inclusão dos artigos que se encaixavam nos padrões desta revisão.

As principais informaçóes sobre os artigos selecionados foram demonstradas por meio de um quadro sinóptico, contendo os seguintes elementos: autor, características da amostra, tipo de intervenção, objetivos principais e desfechos significativos, com exceção do estudo observacional eleito. A análise e a descrição dos dados foram realizadas de forma qualitativa.

As etapas do processo metodológico

Figura 1 - Fluxograma de busca dos artigos

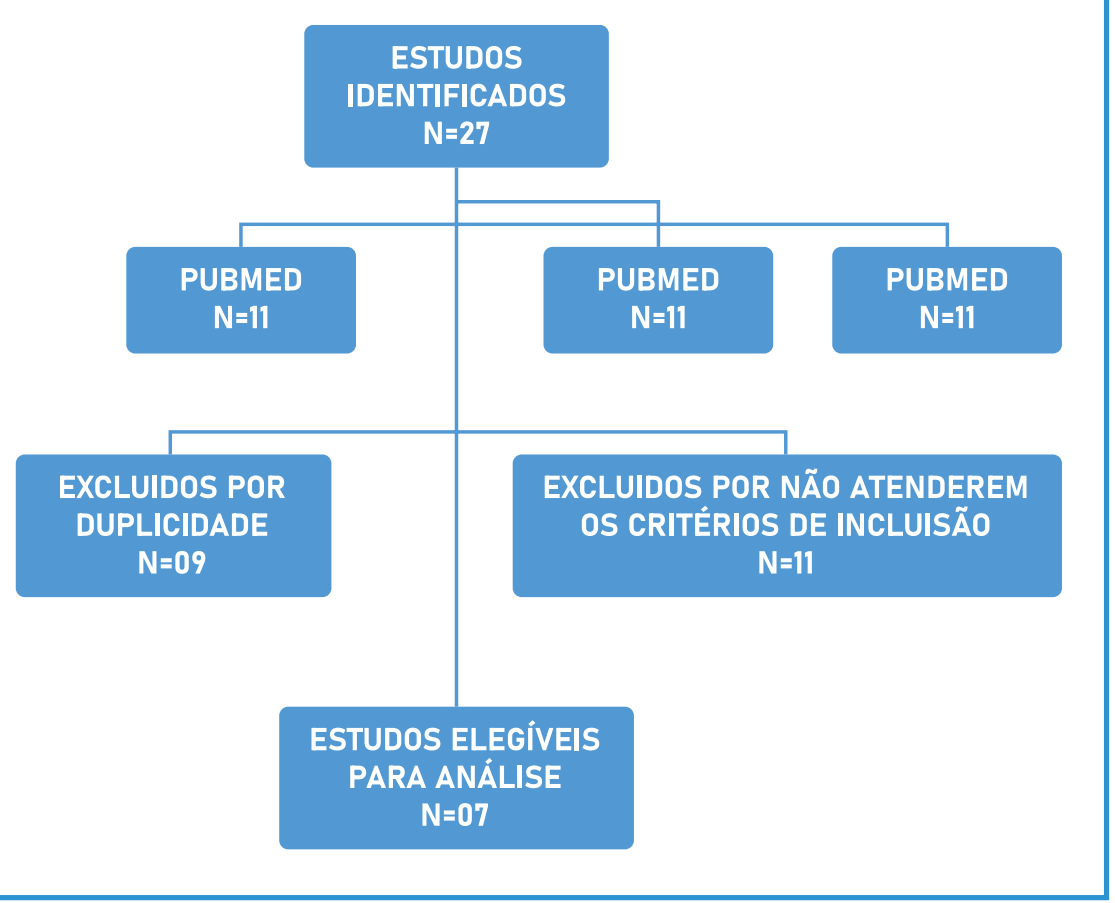

Fonte: Dados da Pesquisa, 2020. descrito encontram-se representadas por meio de um fluxograma (Figura 1).

Foi utilizado um instrumento validado por Ursi ${ }^{(13)}$, para analise e coleta dos dados dos artigos selecionados, a fim de estruturar os resultados e eleger os dados mais significativos. $\mathrm{O}$ instrumento apresenta os seguintes itens: identicação do artigo, instiruição sede do estudo, tipo de publicação, características metodológicas do estudo e avaliação do rigor metodológico $^{(13)}$. Este estudo encontrou relevância em destacar em um quadro esquemático a característica da amostra, o tipo de intervenção utilizada, o objetivo principal do estudo e os desfechos significativos, conforme Quadro 1.

\section{RESULTADOS}

A pesquisa nas bases de dados resultou em 27 artigos, dos quais 20 artigos foram excluídos, devido ao não preenchimento dos critérios de elegibilidade desta revisão ou por apresentarem duplicidade em bases de dados (Figura 1).

A presente revisão contemplou a inclusão de sete artigos, dos quais três eram ensaios clínicos, três eram estudos randomizados controlados e um era estudo observacional. Todos os estudos tiveram como objetivo principal entender a efetividade da acupuntura e algumas de suas variações (a acupressão, moxabustão e a eletroacupuntura) em reduzir as dores do trabalho de parto (Quadro 1).

Em relação ao método de avaliação da intensidade da dor, a maioria dos artigos ${ }^{(5)}$ utilizou como ferramenta uma Escala Visual Analógica (EVA), a qual é uma forma subjetiva de classificação. Além disso, o uso ou não de analgésicos, principalmente a meperidina e a analgesia peridural, também foi empregado como meio de analisar a potência da dor.

Os estudos selecionados também avaliaram outras variáveis relacionadas à acupuntura e o trabalho do parto, como: duração da fase ativa do trabalho de parto, quantidade de ocitocina administrada, efeitos adversos e a satisfação das pacientes.

Verificou-se que, dentre os artigos, o principal acuponto estimulado foi o 


\section{artigo}

Pombo, T.; Costa, A.C.C.; Ferreira, F.S.; Cardoso, S.M.; Ribeiro, R.; Mendes, T.P.L.C.; Antunes, M.B

Acupuntura como método alternativo de analgesia durante parto: uma revisão integrativa

Sanyinjiao, conhecido como Baço-Pâncreas $(\mathrm{Bp}$ 6), responsável pela redução da dor forte durante as contrações uterinas. Este ponto está localizado na parte interna da perna, $9,9 \mathrm{~cm}$ acima da parte superior do tornozelo, logo atrás do osso (figura 2) ${ }^{(14)}$.

Dos estudos examinados, somente um concluiu que não houve benefício analgésico com o uso da acupuntura para o alívio da dor do parto a termo em nulíparas ${ }^{(7)}$.

\section{Figura 2: Acuponto BP6}
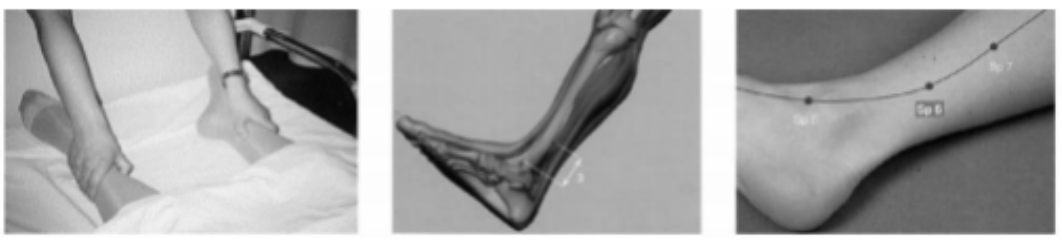

Fonte: Mi-Kyeong Lee, 2003'13

Todos os outros artigos constataram que a acupuntura tem efeito analgésico sobre as dores do parto.

Foi possível determinar também, que a acupuntura tem efeito redutor na duração da fase ativa do trabalho de parto e na quantidade de ocitocina administrada ${ }^{(15,16)}$. A satisfação das pacientes em ter feito uso da acupuntura como analgesia do parto e a intenção de receber acupuntura em partos futuros também foram avaliadas por alguns artigos. A maioria das parturientes mostrou-se satisfeitas com o uso da acupuntura como alívio das dores do parto e também se revelaram positivas em receber acupuntura nas gestações seguintes ${ }^{(1,16)}$.

Quadro 1 - Resumo dos estudos que utilizaram a acupuntura para a analgesia durante o parto. Maringá, PR Brasil, 2020.

\begin{tabular}{|c|c|c|c|c|c|}
\hline ESTUDOS & $\begin{array}{l}\text { AUTOR } \\
\text { PRINCIPAL }\end{array}$ & AMOSTRA & INTERVENÇÃO & OBJETIVO PRINCIPAL & DESFECHOS SIGNIFICATIVOS \\
\hline ESTUDO 1 & $\begin{array}{l}\text { Britt-Ingjerd } \\
\text { Nesheim } \\
\text { (2003) }\end{array}$ & $\begin{array}{l}\text { Parturientes } \\
\text { a termo } \\
N=198\end{array}$ & $\begin{array}{l}\text { Um grupo recebeu acu- } \\
\text { puntura e outro não }\end{array}$ & $\begin{array}{l}\text { Avaliar a efetividade da } \\
\text { acupuntura como analgé- } \\
\text { sico durante } \\
\text { Trabalho de parto. }\end{array}$ & $\begin{array}{l}\text { A acupuntura durante o trabalho } \\
\text { de parto reduziu a necessidade } \\
\text { de outros analgésicos e tem alta } \\
\text { satisfação do paciente } \\
\text { A acupressão por SP6 foi eficaz } \\
\text { para diminuir a dor no parto e di- } \\
\text { minuir o tempo de parto }\end{array}$ \\
\hline ESTUDO 2 & $\begin{array}{l}\text { Mi Kyeong } \\
\text { Lee (2004) }\end{array}$ & $\begin{array}{l}\text { Mulheres } \\
\text { em trabalho } \\
\text { de parto } \\
\mathrm{N}=75\end{array}$ & $\begin{array}{l}\text { Mulheres em trabalho } \\
\text { de parto foram alea- } \\
\text { toriamente designadas } \\
\text { para a acupressão do } \\
\text { SP6 ou grupo de con- } \\
\text { trole de toque SP6 }\end{array}$ & $\begin{array}{l}\text { Avaliar os efeitos da } \\
\text { acupressão SP6 na dor do } \\
\text { parto e no tempo de parto }\end{array}$ & $\begin{array}{l}\text { A acupressão por SP6 foi eficaz } \\
\text { para diminuir a dor no parto e } \\
\text { diminuir o tempo de parto }\end{array}$ \\
\hline ESTUDO 3 & $\begin{array}{l}\text { Sedigheh } \\
\text { Hantoush- } \\
\text { zad h (2007) }\end{array}$ & $\begin{array}{l}\text { Nulíparas } \\
N=144\end{array}$ & $\begin{array}{l}\text { Mulheres nulíparas sau- } \\
\text { dáveis na fase ativa fo- } \\
\text { ram randomizadas para } \\
\text { o estudo e grupo contro- } \\
\text { le, recebendo acupuntu- } \\
\text { ra real e mínima, respec- } \\
\text { tivamente. }\end{array}$ & $\begin{array}{l}\text { Avaliar os efeitos da acu- } \\
\text { puntura em mulheres nu- } \\
\text { líparas durante o trabalho } \\
\text { de parto em relação à dor, } \\
\text { duração e aceitabilidade } \\
\text { materna. }\end{array}$ & $\begin{array}{l}\text { A acupuntura pode reduzir a ex- } \\
\text { periência da dor, a duração da } \\
\text { fase ativa e as unidades de oci- } \\
\text { tocina. Os pacientes foram satis- } \\
\text { feito e nenhum efeito adverso foi } \\
\text { observado. }\end{array}$ \\
\hline ESTUDO 4 & $\begin{array}{l}\text { IZ MacKen- } \\
\text { zie (2011) }\end{array}$ & $\begin{array}{l}\text { Nulíparas a } \\
\text { termo N= } \\
105\end{array}$ & $\begin{array}{l}\text { Estudo duplo-cego de } \\
\text { manual, eletro e simula- } \\
\text { ção acupuntura e estu- } \\
\text { do único-cego compa- } \\
\text { rando acupuntura com } \\
\text { um grupo controle para } \\
\text { analgesia para indução } \\
\text { do parto. }\end{array}$ & $\begin{array}{l}\text { Avaliar o papel da acupun- } \\
\text { tura na analgesia do parto }\end{array}$ & $\begin{array}{l}\text { Não houve benefício analgésico } \\
\text { com o uso da acupuntura para o } \\
\text { alívio da dor do parto a termo em } \\
\text { nulíparas }\end{array}$ \\
\hline
\end{tabular}




\begin{tabular}{|c|c|c|c|c|c|}
\hline ESTUDO 5 & $\begin{array}{l}\text { Jian-mei Cui } \\
\text { (2011) }\end{array}$ & $\begin{array}{l}\text { Primíparas } \\
\text { em trabalho } \\
\text { de parto } \\
N=60\end{array}$ & $\begin{array}{l}\text { As pacientes foram } \\
\text { designadas de acordo } \\
\text { com a sua escolha para } \\
\text { três grupos: } \\
\text { 1- Grupo que recebeu } \\
\text { Mox no SP6 (S-Mox) } \\
\text { 2- Grupo que recebeu } \\
\text { Mox sem ponto de acu- } \\
\text { puntura } \\
\text { 3- Grupo controle que } \\
\text { não receberam Mox }\end{array}$ & $\begin{array}{l}\text { Observar o efeito da moxa- } \\
\text { bustão acuponto Sanyin- } \\
\text { jiao (SP6) (S-Mox) na dura- } \\
\text { ção do primeiro estágio do } \\
\text { trabalho de parto e dor da } \\
\text { contratilidade uterina em } \\
\text { primíparas. }\end{array}$ & $\begin{array}{l}\text { A aplicação do S-Mox poderia } \\
\text { reduzir significativamente a fase } \\
\text { ativa do primeiro estágio do tra- } \\
\text { balho de parto e diminui o esco- } \\
\text { re EVA da dor contrátil uterina, o } \\
\text { que significa aliviando a dor cau- } \\
\text { sada pelo parto vaginal. }\end{array}$ \\
\hline ESTUDO 6 & $\begin{array}{l}\text { Chris Dong } \\
\text { (2014) }\end{array}$ & $\begin{array}{l}\text { Nulíparas } \\
\text { N=180 }\end{array}$ & $\begin{array}{l}\text { As pacientes foram alo- } \\
\text { cadas em dois grupos } \\
\text { experimentais (grupo } \\
\text { EX-B2 e grupo SP6) e } \\
\text { um grupo controle, cada } \\
\text { um com } 60 \text { participantes } \\
\text { elegiveis. }\end{array}$ & $\begin{array}{l}\text { Avaliar os efeitos da eletro- } \\
\text {-acupuntura em diferentes } \\
\text { pontos de acupuntura no } \\
\text { manejo da dor do trabalho } \\
\text { de parto }\end{array}$ & $\begin{array}{l}\text { A aplicação de eletro-acupuntura } \\
\text { nos pontos de acupuntura EX-B2 } \\
\text { SP6 pode ser usado como método } \\
\text { não farmacológico para reduzir a } \\
\text { dor no parto e encurtar a duração } \\
\text { da fase ativa do trabalho de parto. }\end{array}$ \\
\hline
\end{tabular}

\section{DISCUSSÃO}

A dor do trabalho de parto depende de múltiplos fatores e está interligada às características individuais de cada mulher. Para avaliar sua intensidade, os ensaios clínicos analisados lançaram mão dos seguintes recursos: quantidade de analgésicos utilizados intraparto (meperidina e anestesia peridural $)^{1}$, duração da fase ativa do parto ${ }^{2}$ e quantidade de ocitocina utilizada ${ }^{3}$. Além desses, foram utilizadas escalas, um método mais palpável. Nesse sentido, a escala mais utilizada fora a Escala Visual Analógica $(\mathrm{EVA})^{(6,14,17)}$. Além dela, Martini utilizou a simples escala de zero a dez como forma de estimar a dor das parturientes ${ }^{(12)}$.

Neshein verificou que fazer o uso de acupuntura placebo seria uma prática considerada muitas vezes utópica, pois exige habilidades irracionais dos acupunturistas para que conseguissem fazer os pacientes realmente acreditarem que estão recebendo acupuntura. Fato este, que pode alterar significativamente a avaliação da analgesia por acupuntura ${ }^{(1)}$.

Dois estudos analisaram o uso da acupuntura em sua forma básica, por meio de agulhas nos acupontos durante o TP. Tanto Neshein, quanto Hantoushzadeh, concluíram que a acupuntura pode reduzir a experiência da dor do parto e também obteve alta satisfação das pacientes ${ }^{(1,16)}$.
Em contraponto a todos os outros estudos avaliados, um estudo randomizado controlado realizado em uma unidade obstétrica do Reino Unido com 105 nulíparas, concluiu que não houve benefício analgésico com o uso da acupuntura para o alívio da dor do $\mathrm{TP}^{(7)}$. Porém, o único parâmetro utilizado para aferir a dor foi a parcela de gestantes que recorreram a analgesia peridural. $\mathrm{O}$ estudo não foi capaz de analisar o aumento do uso de ocitocina, bem como não utilizou a EVA para conhecer o percentual da dor individual de cada mulher.

Além da acupuntura, alguma das suas principais variações (acupressão, eletroacupuntura e moxabustão - acupuntura térmica) também foi empregada nos estudos analisados. No estudo executado por Kyeong Lee, os efeitos analgésicos de acupressão na dor do parto duraram pelo menos sessenta minutos no grupo que de fato recebeu acupressão no ponto SP6, concluindo que seja improvável que este se trata de um efeito placebo, uma vez que o grupo controle que recebeu apenas o toque no ponto SP6 não apresentou maior redução na dor ${ }^{(14)}$.

No estudo que diz respeito à eletroacuputura, notou-se diferença significativa entre os grupos controle e estudo em termos de alívio da dor durante a fase ativa do trabalho de parto, efeitos que podem ser explicados principalmente pela estimulação e liberação local de endorfinas ao bloquear os impulsos da dor no cérebro, conhecido como a teoria dos portôes ${ }^{(6)}$.

Seguindo a mesma linha, o estudo de Jian-mei Cui revela que aplicação do método de moxabustão S-Mox, poderia reduzir significativamente o tempo da fase ativa do primeiro estágio do TP e diminuir o escore EVA da dor uterina ${ }^{(17)}$.

Esta revisão bibliográfica encontrou relevância em analisar também um estudo observacional, trabalho esse que buscou responder a seguinte pergunta: "As mulheres, durante trabalho de parto normal, em um ambiente hospitalar que recebem acupuntura requerem menos analgesia peridural do que aquelas que não recebem acupuntura?”. A resposta encontrada foi que, de fato, o uso da acupuntura foi associado a um uso reduzido de analgesia peridural. Estavam associados a um aumento do uso de analgesia peridural: ser ocidental, nulípara e ter um parto induzido. Resultado esse que vai de encontro aos frutos desta presente revisão bibliográfi$\mathrm{ca}$, uma vez que o estudo observacional revela que características individuais de cada mulher interferem em maior utilização de analgesia peridural nas parturientes ${ }^{(18)}$.

A revisão dos estudos proporciona um olhar panorâmico acerca do movimento de resgate da naturalização do parto, uma 


\section{artigo}

Pombo, T.; Costa, A.C.C.; Ferreira, F.S.; Cardoso, S.M.; Ribeiro, R.; Mendes, T.P.L.C.; Antunes, M.B.

Acupuntura como método alternativo de analgesia durante parto: uma revisão integrativa

vez que o número de mulheres que optam por terem seu parto natural vem sendo crescente, o que não acontecia anteriormente, principalmente, pela pouca empregabilidade de alternativas que cessassem as dores durante o TP.

Isso se deve a um longo processo, tanto de progresso da medicina, como de crescimento do empoderamento feminino que, cada vez mais, enxerga o parto normal como representação da força que tem uma mulher. O presente trabalho constitui uma ferramenta de comprovação dos efeitos da acupuntura como forma de auxiliar, encorajar e convencer as mulheres de que é possível ter um parto com pouca dor, de forma consistente, embasada por pesquisas reais e sérias empregadas em todo o mundo.

Muitos dos avanços alcançados em ou- tros países contribuem para uma compreensão da questão no Brasil. No entanto, o assunto ainda é pouco difundido em nosso país, o que enfatiza a importância dessa revisão ao trazer essa discussão para a língua portuguesa.

Os estudos aqui analisados, em sua maioria, utilizaram-se da Escala Visual Analógica (EVA), que é um método de avaliação da dor subjetivo, mas que confere confiabilidade aos resultados ${ }^{(19)}$. Vale ressaltar, que pesquisas que se utilizam de experiências pessoais sempre estarão sujeitas a alguns problemas, entre eles, o viés de memória, a supervalorização do fato ocorrido, medo, inibição, entre outros aspectos ${ }^{(20)}$.

Algumas limitações para a construção desta revisão podem ser enumeradas. Em primeiro lugar, embora o tema seja bastan- te debatido e divulgado na mídia leiga, há um número limitado de estudos científicos publicados a respeito do assunto. Em segundo lugar, há uma restrição considerável em como avaliar a dor, por ser uma questão que é muito subjetiva e individual.

\section{CONCLUSÃO}

A acupuntura e suas principais variações abordadas, acupressão, eletroacupuntura e moxabustão - acupuntura térmica, demonstram potencial para contribuir no alívio das dores do trabalho de parto, tanto em sua intensidade quanto em sua duração. Além disso, esse método é capaz de aliviar o sofrimento mental das pacientes, por isso é um instrumento que presta um olhar holístico das parturientes.

\section{REFERÊNCIAS}

1. Neshein BI, Kinge R, Berg B, Alfredsson B, Allgot E, Hove G, Jonhsen W, Jorsett I, Skei S, Solberg S. Acupuncture during labor can reduce the use of meperidine: a controlled clinical study. The Clinical Journal of Pain. 2003;19(3):187-91.

2. Cintra MER, Figueiredo R. Acupuncture and health promotion: possibilities in public health services. Interface - Comunic., Saude, Educ., 2010; 14(32): 39-54.

3. Maike SRL. Fundamentos Essenciais da Acupuntura Chinesa. São Paulo: İcone; 1995.

4. Medeiros R, Saad M. Acupuntura: efeitos fisiológicos além do efeito placebo. O Mundo da Saúde São Paulo: 2009;33(1):69-72.

5. NIN. Painel de Desenvolvimento de consenso do NIN sobre acupuntura - Conferência de 1998. JAMA. 1998; 280 (17):1518-1524.

6. Dong C, Hu L, Liang F, Zhang S. Effects of electro-acupuncture on labor pain management. Archives of Gynecology and Obstetrics; 2014, 291(3):531-536.

7. Mackenzie Iz, Xu J, Cusick C, Midwinter-Morten $\mathrm{H}$, Meacher $\mathrm{H}$, Mollison J, Brock M. Acupuncture for pain relief during induced labour in nulliparae: a randomised controlled study, Epub.2011; 118(4):440-7.

8. WHO. Statement on Caesarean Section Rates. April 2015

9. Kottwitz F, Gouveia HG, Gonçalves A C. Via de parto preferida por puérperas e suas motivações. Escola Anna Nery. 2018; 22(1):e20170013.

10. Tostes NA, Seidl EMF. Expectativas de gestantes sobre o parto e suas percepções acerca da preparação para o parto Temas psicol. 2016; 24(2):681-693.

11. Ruano R, Prohaska C, Tavares AL, Zugaib M. Dor do parto - so- frimento ou necessidade?. Rev. Assoc. Med. Bras. 2007; 53(5): 384. 12. Martini JG, Becker SG. A acupuntura na analgesia do parto: percepções das parturientes. Esc Anna Nery Rev Enferm 2009; 13 (3) 589-594.

13. Ursi ES, Galvão CM. Prevenção de lesões de pele no perioperatório: revisão integrativa da literatura. Rev Latino-am Enfermagem 2006 janeiro-fevereiro; 14(1):124-31.

14. Mi-Kyeong Lee. Effects of San-Yin-Jiao (SP6) Acupressure on Labor Pain, Delivery Time in Women During Labor. Taehan Kanho Hakhoe Chi. 2003; 33(6):753-61.

15. Carvalho AVP, Silva P, Grande AJ. Avaliação do risco de viés de ensaios clínicos randomizados pela ferramenta da colaboração Cochrane. Diagn Tratamento. 2013;18(1):38-44

16. Hantoushzadeh S, Alhusseini N, Lebasch AH. The effects of acupuncture during labour on nulliparous women: a randomised controlled trial. The Australian and New Zealand Journal of Obstetrics \& Gynaecology. 2007; 47(1):26-30.

17. Cui J, Yang X, Jin Z, Ma S, Dong L, Li Q. Effect of Acupoint Sanyinjiao (SP6) Moxibustion on the First Stage of Labor and Uterine Contractive Pain in Primiparae. Chin J Integr Med. 2011:464-466.

18. Kinge R, Neshein BI. Performance of Acupuncture as Labor Analgesia in the Clinical Setting. Acta Obstetricia et Gyneacology Scandinavica. 2006; 85(4):441-3.

19. Mafetoni RR, Shimo AKK. The effects of acupressure on labor pains during child birth: randomized clinical trial. Rev. Latino-Am. Enfermagem. 2016; 24:e2738.

20. KNOBEL, R. Técnicas de acupuntura para alivio da dor no trabalho de parto-Ensaio Clínico. Ensaio Clínico. Campinas, SP : [s.n], 2002 